exposure and the population. Evacuations generally entail moving people through a much higher exposure, albeit for a shorter duration. Our results show that direct exposure to smoke is a more important determinant of ill health than the cumulative exposure to smoke. These results are consistent with other studies. ${ }^{37}$

\section{Reasons for evacuation}

Despite the expert guidance, an unacceptably high proportion of chemical incidents worldwide result in evacuations. Possible reasons include an instinctive response on the behalf of emergency services to evacuate populations in danger, and the preference to "play it safe" by first responders, ${ }^{3}$ and delay in getting appropriate advice. Initial decisions are often taken under very stressful conditions. Lack of experience has also been proposed since greater frequency of evacuations is reported from areas where chemical incidents are uncommon. ${ }^{11}$

We thank the participants in this study for taking the time to complete the questionnaires. We also appreciate the help provided by Geoff Chamings and Shaun Carter at Devon County Council, who converted the postcode references into distance between the factory and the residences.

Contributors: See bmj.com

Competing interests: None declared.

Funding: None.
Ethical approval: Not required at the time. The study was carried out in 1999, when ethics approval was not considered an issue for such studies conducted by health agencies as part of their responsibility.

1 Purdy G, Davies PC. Toxic gas incidents-some important consideration for emergency planning. In: The assessment and control of major hazards. Rugby: Institute of Chemical Engineers, 1985.

2 NHS Executive. Deliberate release of biological and chemical agents: Guidance to help plan the health service response. London: Department of Health, 2000.

3 Essery G. On site emergency planning and the use of predictive techniques.J Loss Prevention in the Process Industries 1991;4:44-8.

Essery G. Chemical incident: evacuate or shelter. Safety Health Practitioner $1994 ; 12: 22-5$.

5 Jones A, Thomson D, Hort M, Devenish BJ. The UK Met Office's next-generation atmospheric dispersion model, NAME III. Proceedings of the NATO CCMS international technical meeting on air pollution modelling and its application (October 2004). Banff, Canada: Kluwer (in press).

6 Morris, RD. Human health effects of a spill of aromatic distillates in Superior, Wisconsin. Atlanta: Agency for Toxic Substances and Disease Registry, Wisconsin. Atlanta: Agency for Toxic

7 Bauer U, Berg D, Kohn MA, Meriwether RA, Nickle RA. Acute effects of nitrogen dioxide after accidental release. Public Health Rep 1998;113: $62-70$

8 Baxter PJ, Heap BJ, Rowland MG, Murray V. Thetford plastics fire, October 1991: the role of a preventative medical team in chemical incidents. Occup Environ Med 1995;52:694-8.

9 Kizer KW, Garb LG, Hine CH. Health effects of silicon tetrachloride. J Occup Med 1984;26:33-6.

10 National Transportation Safety Board. Railroad accident report-Derailment of Illinois central gulf railroad freight train extra 9629 East (GS-2-28) and release of hazardous materials at Livingstone, Louisiana, September 28, 1982. release of hazardous materials at Livingstone, Lou

11 Sorenson JH. Evacuations due to off-site releases from chemical accidents: experience from 1980 to 1984. J Hazardous Materials $1987 ; 14: 247-57$

(Accepted 19 April 2005)

\title{
Commentary: Evacuation decisions in chemical incidents benefit from expert health advice
}

\author{
Peter J Baxter
}

Department of Public Health and Primary Care, University of Cambridge, Institute of Public Health, Cambridge CB2 2SR Peter J Baxter consultant occupational physician Pjb21@medschl. cam.ac.uk
Kinra et al have evaluated symptoms arising from a fire at a plastics factory that lasted 48 hours, in which partial evacuation of the area took place in the first six hours, with most residents remaining indoors for the rest of the emergency. ${ }^{1}$ Statutory emergency planning and advice for people living around designated hazardous installations that manufacture or store chemicals has been based on mathematical modelling of the most likely scenarios for the catastrophic failure of storage vessels or other failures in the plant, in which the duration of flow of a cloud of chemicals that are accidentally released, neutral, or denser than air will be less than 30 minutes (the average time for countermeasures to be implemented). ${ }^{2}$ Peak exposure to an individual living or working nearby at the time when such a chemical cloud disperses should be higher outdoors than inside a building with its doors and windows closed, at least for this short period of time. The difference will depend on how well the building has been sealed against the weather, to reduce normal air infiltration rates. Once the danger has passed, the emergency services would tell people to go outside into the fresh air. Attempts at rapid escape or evacuation are considered to be more dangerous than taking shelter indoors in such short term emergencies, but the adverse health consequences that may follow from this strategy-particularly the effects of exposure to low, cumulative levels of irritant gases in people with asthma and chronic lung disease who do take shelter- need to be studied whenever these unusual incidents occur.

This "stay indoors" strategy may not necessarily apply to certain chemical incidents of longer duration. Smoke from plastics fires usually contains a mixture of highly irritant substances, together with combustion gases, which for polyvinyl chloride (PVC) is mostly hydrogen chloride $(\mathrm{HCl})$. Kinra et al measured 1 part per million of hydrogen chloride in the ambient air of the residential area on their first testing at 12 hours, and thereafter hydrogen chloride and other gases were undetectable. This very soluble gas is unlikely to produce any reactions in people with asthma at this concentration, and healthy individuals can be exposed to higher levels for prolonged periods without ill effects. ${ }^{3}$ Other irritants in the smoke will have an additive effect. Acute incidents involving the inhalation of irritant gases (which are among the most important materials stored at major hazard installations and commonly emitted in fires) may, in severe cases, cause toxic pneumonitis and even death, and brief exposure may trigger reactive airways dysfunction syndrome (RADS; irritant induced asthma). ${ }^{3}$ Kinra et al showed that the adverse respiratory consequences in people with asthma and others were few and concluded that remaining indoors was a safe option in the fire they reported.

Temporary evacuation may nevertheless be advisable where a toxic release is threatened, such as in a 
crash involving a road or rail tanker containing toxic gas. Plumes from burning chemical warehouses, tyre dumps, or plastic stores are usually buoyant from the heat of the fire and may present little immediate risk, but whether they descend to ground level long enough to cause a hazard to the people indoors, and the range at which people could be affected, will depend on the management of the fire by the fire services, the type of materials involved, the wind and weather forecasts, and local topography. ${ }^{4}$ In warehouse fires, chemical fallout from the plume may contaminate nearby gardens and buildings. ${ }^{5}$ As these chemical fires can last for hours, or even days, temporary evacuation when conditions permit should always be considered, ideally with advice provided to the emergency services by a public health response team. ${ }^{4}$ More epidemiological studies with good information on exposure will be essential to build the evidence base for decision making in chemical releases and for management after the incident.

1 Kinra S, Lewendon G, Nelder R, Herriott N, Mohan R, Hort M, Harrison $\mathrm{S}$, Murray V. Evacuation decisions in chemical air pollution incidents: cross sectional survey. BMJ 2005;330:1471-4.

2 Health and Safety Executive. Control of major accident hazards (COMAH). www.hse.gov.uk/comah/index.htm (accessed 31 May 2005).

3 Department for Environment, Food and Rural Affairs. Expert Panel on Air Quality Standards. Guidelines for halogens and hydrogen halides in ambient air for protecting human health against acute irritancy effects. Comsula ent air for protecting human health against acute irritancy effects. Consultation document. London: Defrc ity/aqs/index.htm

4 Baxter PJ, Heap BJ, Rowland MGM, Murray VSG. Thetford plastics fire, October 1991: the role of a preventative medical team in chemical incidents. Occup Environ Med 1995;52:694-8

5 Baxter PJ. Review of major chemical incidents and their medical management. In: Murray V, ed. Major chemical disasters-medical aspects of management. London: Royal Society of Medicine, 1990:7-20. (Royal Society of Medicine Services. International Congress and Symposium Series No 155.)

\section{The role of healthcare delivery in the outcome of meningococcal disease in children: case-control study of fatal and non-fatal cases}

Nelly Ninis, Claire Phillips, Linda Bailey, Jon I Pollock, Simon Nadel, Joseph Britto, Ian Maconochie, Andrew Winrow, Pietro G Coen, Robert Booy, Michael Levin

\begin{abstract}
Objective To determine whether suboptimal management in hospital could contribute to poor outcome in children admitted with meningococcal disease.

Design Case-control study of childhood deaths from meningococcal disease, comparing hospital care in fatal and non-fatal cases.

Setting National statistics and hospital records. Subjects All children under 17 years who died from meningococcal disease (cases) matched by age with three survivors (controls) from the same region of the country.

Main outcome measures Predefined criteria defined optimal management. A panel of paediatricians blinded to the outcome assessed case records using a standardised form and scored patients for suboptimal management.

Results We identified 143 cases and 355 controls. Departures from optimal (per protocol) management occurred more frequently in the fatal cases than in the survivors. Multivariate analysis identified three factors independently associated with an increased risk of death: failure to be looked after by a paediatrician, failure of sufficient supervision of junior staff, and failure of staff to administer adequate inotropes. Failure to recognise complications of the disease was a significant risk factor for death, although not independently of absence of paediatric care $(\mathrm{P}=0.002)$. The odds ratio for death was $8.7(95 \%$ confidence interval 2.3 to 33 ) with two failures, increasing with multiple failures.

Conclusions Suboptimal healthcare delivery significantly reduces the likelihood of survival in
\end{abstract}

children with meningococcal disease. Improved training of medical and nursing staff, adherence to published protocols, and increased supervision by consultants may improve the outcome for these children and also those with other life threatening illnesses.

\section{Introduction}

Although treatment of meningococcal disease on a paediatric intensive care unit improves outcome, ${ }^{12}$ most patients present to their nearest emergency department and many deteriorate so rapidly that death from shock and multiorgan failure often occurs before transfer to a specialist paediatric intensive care unit. The speed with which the diagnosis is made, antibiotics administered, and the complications of shock and multiorgan failure treated is likely to be a major determinant of outcome. ${ }^{3}$ To test the hypothesis that outcome depends on the quality of health care early in the disease we undertook a national, blinded, case-control study of healthcare delivery in the first 24 hours after admission to hospital in children who died from meningococcal disease compared with those who survived.

\section{Methods}

We identified cases of meningococcal disease in children aged 0-16 years between 1 December 1997 and 28 February 1999. For each death (case) we identi-
Infectious Diseases Unit, Department of Paediatrics, Faculty of Medicine, Imperial College of Science, Technology and Medicine, London W2 1PG

Nelly Ninis

clinical research fellow Michael Levin professor of paediatric infectious diseases

Research Unit, Royal College of

Paediatrics and

Child Health,

London W1W 6DE

Claire Phillips

research assistant

Linda Bailey research nurse

Faculty of Health and Social Care, University of the West of England, Bristol BS16 1DD Jon I Pollock principal lecturer in epidemiology

continued over

BMJ 2005;330:1475-8 